\title{
LA REFORMA CONSTITUCIONAL EN MATERIA DE DERECHOS HUMANOS DE 2011: EL CAMBIO DE PARADIGMA QUE AÚN NO TERMINA DE SER
}

OMAR GARCÍA LUNA*

\section{RESUMEN}

En el presente trabajo se analiza la forma en que ha sido interpretada la reforma constitucional en materia de derechos humanos de 2011 por parte de los tribunales federales, en particular, por la Suprema Corte de Justicia de la Nación. A través del análisis de distintas tesis jurisprudenciales se argumenta que la Suprema Corte se ha constituido en un elemento de resistencia frente al cambio de paradigma propuesto en la reforma al artículo primero constitucional.

\section{Palabras clave:}

Derechos humanos, Jurisprudencia, Tribunales federales, Artículo primero constitucional

\section{ABSTRAC}

This paper analyzes the way in which the 2011 constitutional reform on human rights has been interpreted by the federal courts, in particular, by the Supreme Court of Justice of the Nation. Through the analysis of different jurisprudential theses it is argued that the Supreme Court has become an element of resistance against the change of paradigm proposed in the first constitutional article.

\section{Keywords:}

Human rights, Jurisprudence, Federal courts, First constitutional article

\section{INTRODUCCIÓN}

El 10 de junio de 2011 se publicó en el Diario Oficial de la Federación la que ha sido considerada como una de las reformas más ambiciosas y trascendentes en

* Universidad Anáhuac, garcialuna_omar@yahoo.com.mx 
la historia de la actual Carta Magna. ${ }^{1}$ Por efectos de esta, los tratados internacionales en materia de derechos humanos deben ser considerados, sin lugar a duda, como parte integral del orden jurídico nacional, al menos al mismo nivel jerárquico que la propia constitución; además, se fijaron una serie de principios que tienen por objeto asegurar que estos cambios irradien al resto del sistema. ${ }^{2}$ Por supuesto, se trata de una modificación ambiciosa, al grado que se le ha presentado como un auténtico cambio de paradigma en el sistema jurídico mexicano.

Asumiendo que un cambio de paradigma no puede limitarse a simplemente modificar el texto de un cuerpo normativo, así sea este la propia constitución, sino que debe reflejarse en las prácticas jurídicas cotidianas, a varios años ya de que tuviera lugar esta reforma, parece pertinente formular la pregunta sobre cuál es el avance de la misma; es decir, ¿hasta dónde realmente han mejorado las condiciones de respeto y ejercicio de los derechos humanos en este país?

Ahora bien, debido que esta reforma afecta a prácticamente todos los ámbitos de la vida jurídica e institucional de este país, intentar una respuesta que sea comprehensiva de esta realidad es algo que rebasa los límites de un trabajo como este. En cambio, lo que aquí se propone es una valoración focalizada y cualitativa, concentrada en la forma en que la reforma ha sido interpretada por el Poder Judicial de la Federación, incluyendo la Suprema Corte de Justicia. A todo esto, se debe tener en cuenta que bajo ciertas condiciones los criterios jurisprudenciales emitidos por estos órganos son de observación obligatoria para tribunales y jueces ordinarios, y al mismo tiempo sirven como criterio orientador para las resoluciones que emiten todas las autoridades administrativas. Por esta razón la forma en la que los derechos humanos son interpretados en las tesis jurisprudenciales afecta o se transmite al resto del sistema jurídico e institucional, de ahí el interés por estudiarlas en el presente trabajo.

En este sentido, y sólo con el propósito de contextualizar, en las siguientes líneas se lleva a cabo una breve descripción del contenido de la reforma. De igual forma, a efecto de contar con parámetros de comparación, el análisis no se limita a las tesis jurisprudenciales vertidas de forma posterior a la misma, sino que se estudian algunos criterios relevantes que se adoptaron en el transcurso de las dos décadas pasadas. El propósito de ello es mostrar cómo antes de la reforma el Poder Judicial de la Federación se estaba constituyendo en un claro defensor de los derechos humanos reconocidos a nivel internacional y que, paradójicamente, después de 2011, los altos tribunales parecen estar adoptando criterios menos favorables.

1 En lo sucesivo se hará referencia a este decreto simplemente como la reforma o la reforma de 2011.

2 Con esta reforma también se modificaron otros numerales de la Constitución, destacándose la transformación sufrida en el contenido del artículo 33, con lo cual se eliminó la facultad discrecional a favor del Presidente de la República, mediante la cual, sin necesidad de audiencia previa, podía hacer abandonar el territorio nacional a cualquier extranjero que considerase pernicioso para el país. En el ámbito internacional se consideró reiteradamente que esta facultad del Poder Ejecutivo violaba el derecho de audiencia y el derecho a un juicio justo, por lo que se recomendó al Estado mexicano que la eliminara o modificara. El constituyente optó por mantener esa facultad, pero con la reforma se reconoce el derecho de audiencia a favor del extranjero afectado. En el resto de artículos reformado se introdujeron cambios necesarios con el fin de ajustarlos a lo dispuesto en el artículo primero. 


\section{LA REFORMA: ANÁLISIS AL ARTÍCULO PRIMERO CONSTITUCIONAL}

La reforma en materia de derechos humanos de 2011 tuvo su origen en medio de una grave crisis de violencia y criminalidad. No es este el lugar para resumir ni para tratar de dimensionar circunstancias que son de sobra conocidas. La guerra contra el narcotráfico ha provocado cifras que coloca a México como uno de los países más peligrosos del mundo, con datos de muertes violentas aún mayores que los que se registran en países que están viviendo guerras civiles o internacionales. ${ }^{3}$ Además, si bien los índices de criminalidad en materia de secuestro, extorción, robo y violaciones son apabullantes, desafortunadamente, debido a la cifra negra, estos no terminan de reflejar la realidad. ${ }^{4}$ Lo más graves es que el Estado mismo es el origen de muchas de estas afectaciones, principalmente a través de las fuerzas armadas, policiacas y otras agencias de control. ${ }^{5}$ En consecuencia, se ha generado una fuerte presión internacional y doméstica, para que sean adoptadas providencias de fondo que pongan freno esta situación. Una de estas medidas fue precisamente la reforma en comento, a través de la cual se esperaba provocar un cambio sustancial en la forma en la que actúan las autoridades en todos los órdenes de gobierno; pero también se esperaba incidir en la población, fomentando un mejor conocimiento de los derechos que les son reconocidos a los ciudadanos, así como de los medios de los cuáles disponen para hacerlos valer. En suma, el objetivo era crear una cultura de respeto y protección de los derechos humanos.

Así, el 10 de junio de 2011 se publicó en el Diario Oficial de la Federación una serie de modificaciones a distintos artículos de la Constitución, que en su conjunto se conocen como la reforma en materia de derechos humanos. En concreto se reformaron los artículos $1,3,11,15,18,29,33,89,97,102$ y 105 ; y como ya se ha hecho notar, el eje en torno al cual gira toda la reforma es el artículo primero. ${ }^{6}$ Por ello, debido a su importancia, a continuación se transcribe la parte

3 Muchos son los informes generados por organismos nacionales e internacionales en los que se diagnostica la grave situación que atraviesa México en materia de derechos humanos, en este caso, sólo para efectos de documentación se recomienda consultar el informe elaborado por la Comisión Interamericana de Derechos Humanos en 2015. (Comisión Interamericana de Derechos Humanos, Situación de derechos humanos en México, 2015.

4 Se entiende por cifra negra el porcentaje de delitos que se calcula no son denunciados por las víctimas, ya sea porque no existen a mano las oficina del estado en dónde puedan hacerlo (falta de cobertura institucional), por desconfianza de los resultados que se obtendrán (corrupción o ineficiencia institucional) o por miedo de las represalias que puedan tomar los criminales. Para una percepción inicial pero completa de este fenómeno se puede consultar la siguiente dirección electrónica: http://www.semaforo.mx/content/semaforode-cifra-negra.

5 De acuerdo con datos de la Comisión Nacional de Derechos Humanos, de 2006 a 2017 fueron más de 20 mil las quejas recibidas en contra de miembros del Ejército, Marina y Policía Federal; aun cuando desde 2012 existe una paulatina disminución en los porcentajes de las mismas. Esta cifra no toma en cuenta a las policías estatales y municipales (Cfr., GANDARIA MANRIQUE, "CNDH recaba 20 mil quejas en contra de Ejercito Marina y PF", en El Sol de México, 12 de noviembre de 2017).

6 Para una síntesis completa del proceso legislativo y de las oposiciones partidarias que sufrió la reforma en materia de derechos humanos se recomienda consultar a RODRIGO LABARDINI, "Proteo en México. Un 
conducente, contrastándola con el texto anterior. Posteriormente se explica el alcance de las modificaciones introducidas.

Texto anterior:

ArTículo 1o. En los Estados Unidos Mexicanos todo individuo gozará de las garantías que otorga esta Constitución, las cuales no podrán restringirse ni suspenderse, sino en los casos y con las condiciones que ella misma establece. (...)

Texto actual (Diario Oficial de la Federación de 10 de junio de 2011):

ARTículo 1o. En los Estados Unidos Mexicanos todas las personas gozarán de los derechos humanos reconocidos en esta Constitución y en los tratados internacionales de los que el Estado Mexicano sea parte, así como de las garantías para su protección, cuyo ejercicio no podrá restringirse ni suspenderse, salvo en los casos y bajo las condiciones que esta Constitución establece.

Las normas relativas a los derechos humanos se interpretarán de conformidad con esta Constitución y con los tratados internacionales de la materia favoreciendo en todo tiempo a las personas la protección más amplia.

Todas las autoridades, en el ámbito de sus competencias, tienen la obligación de promover, respetar, proteger y garantizar los derechos humanos de conformidad con los principios de universalidad, interdependencia, indivisibilidad y progresividad. En consecuencia, el Estado deberá prevenir, investigar, sancionar y reparar las violaciones a los derechos humanos, en los términos que establezca la ley.

(...)

El primer cambio que salta a la vista es la introducción de la expresión "derechos humanos"; la cual sustituye a la noción de "garantías individuales" como protagonista del texto, aunque no la elimina. De entrada, esto no debe ser visto como de un mero cambio en la denominación; por principio de cuentas representa la incorporación de todo el cuerpo de derecho internacional protector de derechos humanos en el sistema jurídico nacional, pero además se sustituye una concepción decimonónica de los derechos por otra más moderna, al tiempo que se integra una nueva visión del papel que tiene el Estado en la protección de los mismos. $^{7}$

Se pueden mencionar al menos dos buenas razones para distinguir entre las nociones de "garantías individuales" y "derechos humanos". La primera, de carácter más bien teórico, parte del punto que la expresión "garantías individuales"

nuevo paradigma: derechos humanos y constitución", en Boletín Mexicano de Derecho Comparado, No. 133, Enero abril de 2012, pp. 319-353.

7 El debate sobre la diferencia terminológica y conceptual entre las expresiones derechos humanos, garantías individuales y otras parecidas es algo que está lejos de concluir. La mayoría de los estudiosos de la cuestión adopta soluciones y posturas distintas dependiendo de los fundamentos teóricos de los cuales parta. Sin embargo, si sobre algo existe acuerdo es en el hecho de que la reforma, más allá de ser un mero cambio terminológico, lo que intenta es introducir una forma más moderna y amplia de concebir los derechos de los ciudadanos. Al respecto, puede consultarse RAMÓN GIL CARREÓN GALLEGOS, "Derechos humanos, garantías individuales y derechos fundamentales. Problema terminológico o conceptual", en DAVID CIENFUEGOS SALGADO y German FROTO MANDARIAGA (Coord.), Los derechos humanos en el momento actual, Comisión de Derechos Humanos de Coahuila, 2012, pp. 131-145. 
denota una postura iuspositivista, desde la cual se considera que los derechos del hombre son creados y otorgados por el orden jurídico. Por esta razón, en el anterior texto se afirma que tales garantías las "otorga esta Constitución". En otras palabras, dentro del texto constitucional se inserta una teoría iuspositivista; conforme a la cual, los individuos no poseen más derechos que los que el propio Estado, a través del orden jurídico, quiera o está en capacidad de concederles y garantizar. En cambio, parece claro que con la expresión "derechos humanos", la Constitución adopta un trasfondo de corte más cercano al iusnaturalismo. Esto es, acepta que las personas poseen derechos previos e independientes al Estado y que al sistema jurídico le corresponde simplemente reconocer tales derechos. De ahí el cambio de la palabra "otorga" por "reconoce". ${ }^{8}$ Un argumento más simple, pero no banal que puede explicar la elección de esta denominación, es que se aprovechó que en los instrumentos internacionales relativos, la expresión que se ocupa es la de "derechos humanos"; por lo tanto, al incorporar todo ese cuerpo normativo en el derecho nacional, lo recomendable fue seguir usando la misma terminología. ${ }^{9}$

Aun con todo, en la nueva redacción se conserva la figura de "garantías", pretendiendo con ello establecer una distinción entre los auténticos "derechos" y los mecanismos que el Estado está obligado a crear para hacer válidos o garantizar los primeros. El problema con esta redacción no es sólo que se mantiene dentro del texto constitucional una expresión arcaica, aun cuando se pretende con significado distinto; sino que se consagra una distinción que no tiene sentido. Se debe tomar en cuenta que si una "garantía" se encuentra consagrada en la constitución o en un tratado internacional no es un mero mecanismo para hacer efectivo otro derecho, sino que en sí mismo debe ser considerado un derecho, de forma más precisa un derecho humano. Y por efecto del principio pro persona, contenido en el segundo párrafo del artículo primero constitucional, se puede decir lo mismo respecto de las leyes secundarias; las cuales pueden prevalecer sobre la constitución o sobre los tratados internacionales en el caso de que ofrezcan mejores garantías que estos instrumentos.

Todo ello provoca que a jueces y tribunales, se les otorgue en papel más activo en la integración del sistema jurídico, ya que la responsabilidad de aplicar el contenido de los tratados internacionales recae en última instancia en ellos. De esta manera, están obligados a subsanar las posibles lagunas y contradicciones del sistema jurídico nacional, en tanto deben interpretarlo de conformidad a lo que se establece en los tratados en materia de derechos humanos. De esta manera, los jueces pasan de ser simples aplicadores de la norma a ser verdaderos creadores de la misma ${ }^{10}$.

8 Adoptar o desprender una posición teórica del contenido de una constitución es una cuestión que puede tener enorme relevancia incluso para efectos prácticos. En este sentido puede consultarse a CRUZ PARCERO JUAN ANTONIO, quien explica esta cuestión en su obra Hacía una Teoría Constitucional de los Derechos Humanos, (Instituto de Estudios Constitucionales del Estado de Querétaro, México, 2017).

9 Se ha señalado que la expresión "derechos fundamentales" hubiera sido una solución más afortunada (Cfr.)

10 En los últimos años es abundante la literatura en la que se discute y se toma posición (a favor o en contra) en torno al nuevo rol que se supone están adoptando los jueces en los sistemas jurídicos contemporáneos. En términos generales se acepta que existe una tendencia en la legislación y en la forma en la que se autoconciben los jueces, conforme a la cual, la labor judicial se concibe más dinámica y creativa, debido sobre todo a 
De hecho, en la formula introducida en el artículo primero constitucional no sólo los jueces, sino que cualquier autoridad en el ámbito de su competencia, está obligada a llevar a cabo una labor interpretativa de este tipo.

En la parte final del primer párrafo del artículo primero de la Constitución se contiene una disposición que ha sido objeto de controversia. Se trata de una frase, según la cual los derechos humanos reconocidos en los tratados internacionales no podrán suspenderse ni restringirse salvo en aquellos casos en los que la Constitución así lo prevea. La disposición por sí misma es contradictoria del principio según el cual los estados signatarios de un instrumento internacional no pueden oponer normas de derecho interno para desconocer las obligaciones contraídas a nivel internacional. Además, es contraria al principio pro persona, ya que en esos casos evidentemente no podrá aplicarse la norma que más amplia protección ofrezca, debiendo estar a lo que la restricción constitucional indica.

En el segundo párrafo se contienen dos principios fundamentales para la interpretación y aplicación de las normas jurídicas desde la óptica de los derechos humanos: el principio pro persona ${ }^{11}$ y el principio de interpretación conforme. ${ }^{12}$ De acuerdo con el primer principio mencionado, en toda situación jurídica en la que deban aplicarse derechos humanos debe prevalecer el sentido que sea más favorable a la persona. De esta manera, se rompe el principio de jerarquía jurídica estricta pues ahora se debe considerar como norma superior la que mejor protección ofrezca, sea la ley ordinaria, sea un tratado o sea la propia Constitución. Por su parte, el principio de interpretación conforme exige que las normas de derecho nacional deban ser forzosamente interpretadas teniendo en mente siempre el contenido relativo de los tratados internacionales en materia de derechos humanos. Como se puede observar en relación a estos principios, uno a otro se refuerza y potencia.

Del tercer párrafo se desprende que la obligación de promover y proteger los derechos humanos no se limita a los órganos jurisdiccionales, sino que corre a cargo de cualquier autoridad; así mismo, se impone la obligación de llevar a cabo esta tarea atendiendo siempre a los principios de universalidad, interdependencia, indivisibilidad y progresividad. ${ }^{13}$

la relevancia que han tomado las normas tipo principios de redacción abierta general, frente a las normas tipo reglas de redacción concreta y específica; lo cual exige de los intérpretes más una operación de ponderación que de mera subsunción. (Cfr., PEDRO ANTONIO ENRÍQUEZ SOTO, "La interpretación conforme y su impacto en los jueces mexicanos", en José María Serna de la Garza (coord.), Contribuciones al derecho constitucional, UNAM, IIJ, México, 2015, pp. 289-215.).

11 Cfr., JOSÉ LUIS CABALLERO OCHOA, "La cláusula de interpretación conforme y el principio pro persona (artículo primero segundo párrafo de la constitución)", en Miguel Carbonell Sánchez y Pedro Salazar Ugarte (coords.) La reforma constitucional de derechos humanos: un nuevo paradigma, UNAM-IIJ, México, 2011, pp. 103-133.

12 Cfr., EDUARDO FERRER MAC-GREGOR, "Interpretación conforme y control difuso de la convencionalidad. El nuevo paradigma para el juez mexicano", en Miguel Carbonell Sánchez y Pedro Salazar Ugarte (coords.) La reforma constitucional de derechos humanos: un nuevo paradigma, UNAM-IIJ, México, 2011, pp. 339-429.

13 Cfr., LUIS DANIEL VÁZQUEZ y SANDRA SERRANO, "Los principios de universalidad, interdependencia, indivisibilidad y progresividad. Apuntes para su aplicación práctica", en MIGUEL CARBONELL SÁN- 
Se entiende por universalidad el que los derechos humanos son reconocidos a todas y cada una de las personas, sin excepción. Con las nociones de interdependencia e indivisibilidad, se pretende que estos derechos deben ser considerados como una unidad jurídica, por ello cada uno se interpreta en relación a todos los demás. Por último, la progresividad, es una nota que puede tener dos interpretaciones. De acuerdo con la primera se obliga al legislador o constituyente a no introducir restricciones o mermas de los derechos en reformas futuras. A través de la segunda, se reconoce que no todos los derechos pueden ser una realidad para todas las personas al mismo tiempo, en particular en el caso de los derechos económicos y sociales. No obstante, se impone al Estado la obligación de crear las condiciones adecuadas para que cada vez sea mayor el número de personas que gozan de todos (o casi todos) sus derechos. Estos cuatro principios se suman a los dos que se contienen en el párrafo segundo, y todos, en su conjunto, deben ser tomados en cuenta por el intérprete jurisdiccional o administrativo cuando se encuentre en un caso que involucre derechos humanos.

Vale la pena cerrar este apartado insistiendo en que las modificaciones al artículo primero constitucional han tenido como objetivo promover una reforma estructural del Estado mexicano, en otras palabras, pretenden provocar un cambio de paradigma. Y que la cuestión que sirve de hilo conductor al presente trabajo es comprender si tanto la reforma constitucional, como las posteriores interpretaciones del poder judicial realmente permiten considerar que dicho cambio paradigmático se ha producido o al menos está en marcha.

Por el momento se debe reconocer que en los tres primeros párrafos del artículo primero constitucional se presupone una transformación completa, no sólo de la forma en que se integra e interpreta el orden jurídico nacional, sino también de las prácticas que desarrollan jueces y tribunales. En consecuencia, de forma implícita se propone una alteración significativa del juego de contrapesos institucionales derivados de la división de poderes, en consecuencia el Poder Judicial adquiere un papel mucho más relevante frente al legislativo y el ejecutivo. Este último punto ha sido fuertemente criticado por algunos sectores que consideran que estas nuevas facultades trastocan el equilibrio de poderes, ya que se crea un super poder que no fue electo por voto directo. A esta aseveración debe objetarse que por efecto del párrafo tercero del nuevo artículo primero constitucional, es el propio Estado mexicano el que accede a reorganizarse en torno al concepto de derechos humanos; en otras palabras, se asume, de conformidad con lo que indican las teorías políticas más reputadas de la actualidad, que las democracias modernas deben estructurarse en torno al reconocimiento y protección de los derechos humanos de los ciudadanos; ya que estos derechos son la única idea que hoy por hoy logra legitimar la existencia de los Estados. ${ }^{14}$

CHEZ y PEDRO SALAZAR UGARTE (coords.) La reforma constitucional de derechos humanos: un nuevo paradigma, UNAM-IIJ, México, 2011, pp.135-165.

14 Cfr., JÜRGEN HABERMAS, Facticidad y validez. Sobre el derecho y el estado social de derecho en términos de teoría del discurso, Trotta, Madrid, 4a ed. 1998; JOHN RAWLS, Teoría de la justicia, Fondo de Cultura Económica, México, 2010. 


\section{LOS DERECHOS HUMANOS EN LOS CRITERIOS JURISPRUDENCIALES ANTES DE LA REFORMA DE 2011}

Como se puede desprender de lo que se ha dicho con anterioridad, cuando se habla de un cambio de paradigma se presume la existencia de un antes y un después en la situación o problema de estudio. Así, con el fin de poder disponer de un parámetro de comparación, en el presente apartado se revisan los criterios que han vertido los tribunales de la federación en relación a los tratados internacionales en los que se establecen derechos humanos, antes y después de la reforma de 2011.

La que resulta, a partir de la última década del siglo pasado y la primera del presente, es una historia de apertura paulatina por parte de los tribunales federales a favor de los derechos humanos, lo cual hacía pensar que se les podía considerar como agentes promotores de una "revolución de los derechos", ${ }^{15}$ aún antes de la reforma de 2011. En contraste, de forma reciente se han adoptado criterios que dejan en suspenso todo el proceso; al grado que se puede decir que por vía jurisdiccional la Suprema Corte de Justicia está dejando sin efectos la mencionada reforma.

En la jurisprudencia del Poder Judicial de la Federación, el problema se entreteje en torno a dos cuestiones estrechamente relacionadas; la primera es el problema de la jerarquía normativa de los tratados internacionales en el derecho mexicano; la segunda, es la posibilidad de hacer valer los tratados internacionales en derechos humanos dentro de una argumentación jurídica.

La cuestión de la jerarquía normativa es de suma importancia en el manejo de un orden jurídico, pues es uno de los criterios principales que permite dirimir una controversia en caso de que diferentes normas entren en contradicción. Así, un criterio de superioridad permite que una de las normas subsista y que la otra eventualmente sea excluida ${ }^{16}$. Debido a que el trabajo de los cuerpos legislativos no es lo expedito que se quisiera, sobre todo porque funciona conforme a tiempos políticos, se considera que es más ágil acudir a tribunales superiores que puedan dirimir la controversia a través de sus resoluciones, y de esta manera ir depurando el sistema eliminando posibles contradicciones.

En el caso del derecho mexicano el criterio principal para establecer la jerarquía normativa se encuentra en el artículo 133 constitucional; el cual, no fue objeto de la reforma de 2011 en materia de derechos humanos. Este artículo, desde que fue promulgada la actual Carta Magna sólo ha sido reformado en dos ocasiones, la primera en 1934 y la última en 2016. La primera de estas reformas (la de 1934) se promovió debido a que se consideraba que la redacción original de este numera dejaba lugar a una interpretación en la que los tratados internacionales y la propia Constitución pudieran estar en la misma jerarquía

\footnotetext{
15 Para usar la expresión acuñada por Charles Epp (La revolución de los derechos. Abogados, activistas y cortes supremas en perspectiva comparada, Siglo XXI, México, 2013)

16 Cfr., CARLOS M. AYALA CORAO, La jerarquía constitucional de los tratados relativos a derechos humanos y sus consecuencias, Colegio de Secretarios de la Suprema Corte de Justicia de la Nación, México, 2003.
} 
normativa, ${ }^{17}$ posibilidad que para aquella época se consideraba inaceptable; razón por la cual se decidió modificar este artículo y establecer claramente que los tratados internacionales sólo podían considerarse parte del sistema jurídico nacional en tanto "estuvieran de acuerdo con la misma"; es decir, con la Constitución.

Siendo que el texto del artículo 133 constitucional que se adoptó en 1934 seguía estando en vigor para 2011, fecha en que se promulgó la reforma en materia de derechos humanos, entonces, al menos por el momento conviene tener presente dicho texto, y no el que resultó de la reforma de 29 de enero de $2016 .{ }^{18}$ A continuación se transcribe el texto del artículo 133 constitucional vigente en 2011:

\begin{abstract}
ARTículo 133. Esta Constitución, las leyes del Congreso de la Unión que emanen de ella y todos los tratados que estén de acuerdo con la misma, celebrados y que se celebren por el Presidente de la República, con aprobación del Senado, serán la Ley Suprema de toda la Unión. Los jueces de cada Estado se arreglarán a dicha Constitución, leyes y tratados, a pesar de las disposiciones en contrario que pueda haber en las Constituciones o leyes de los Estados.
\end{abstract}

El contenido de este numeral ha sido acusado de mostrar un cierto grado de ambigüedad, pues si bien siempre se consideró que no había o no podía haber duda acerca de la supremacía de la Constitución dentro del sistema jurídico nacional; no obstante, de la simple lectura no se desprende un criterio claro de superioridad o igualdad entre las leyes federales y los tratados internacionales. ${ }^{19} \mathrm{De}$ manera que ha sido necesario acudir a los tribunales federales, para que, determinen lo conducente. Para efectos de este trabajo no es necesario retrotraerse demasiado en el tiempo; por lo cual, basta con tener presente cuales han sido los criterios sostenidos por el poder judicial de la federación desde la década de los noventa del siglo pasado. En ese entonces, el criterio dominante era que las leyes federales y los tratados internacionales se encuentran en la misma jerarquía normativa; si bien ambos por debajo de la Constitución. A efecto de que sean evidentes los contrastes que se quieren hacer notar a continuación se trascribe el texto de esta tesis jurisprudencial:

\title{
LEYES FEDERALES Y TRATADOS INTERNACIONALES TIENEN LA MISMA JERARQUIA NORMATIVA.
}

De conformidad con el artículo 133 de la Constitución, tanto las leyes que emanen de ella como los tratados internacionales, celebrados por el Ejecutivo Federal, aprobados por el Senado de la república y que estén de acuerdo con la misma, ocupan, ambos, el rango inmediatamente inferior en la jerarquía de las normas en el orden jurídico mexicano. Ahora bien, teniendo la misma jerarquía, el tratado internacional no puede ser criterio para determinar la constitucionalidad de una ley

$\overline{17}$ En la redacción original de 1917 no se hacía mención de que, para poder ser considerados porte integral del derecho mexicano, los tratados internacionales debían estar de acuerdo con la Constitución.

18 De cualquier modo, para efectos de este trabajo la reforma de 29 de enero de 2016 no es trascendental, ya que únicamente se sustituyó la expresión "Estados" por "entidades federativas"; manteniéndose el mismo texto en lo sustancial.

19 Una breve síntesis del proceso jurisprudencial de conceptualización de los derechos humanos en los criterios jurisprudenciales del Poder Judicial de la federación es ofrecida por AYALA CORAO, op., cit. 
ni viceversa. Por ello, la Ley de las Cámaras de Comercio y de las de la Industria no puede ser considerada inconstitucional por ser contrario a lo dispuesto en un tratado internacional. ${ }^{20}$

A lo largo de la segunda mitad del siglo pasado México fue un activo promotor del sistema interamericano y del sistema internacional de derechos humanos, y para la década de los noventa ya tenía suscritos un número considerable de este tipo de instrumentos; los cuales, al ser suscritos por el Ejecutivo y ratificados por el Senado, debían ser considerados como parte del orden jurídico nacional. Ahora bien, de acuerdo con la tesis recién transcrita, los tratados internacionales y las leyes secundarias debían ser considerados en el mismo nivel jerárquico normativo, en consecuencia, los tratados no podían ser usados para efectuar control de legitimidad normativa sobre las leyes ordinarias. Aun cuando las leyes ordinarias fueran contrarias a las obligaciones contenidas en tratados internacionales, no era posible argumentar que dichas leyes no debían de ser aplicadas por ser contrarias a tratados internacionales. Por esta razón, en la práctica carecía de sentido recurrir a los tratados internacionales para hacer valen un argumento en contra de una ley violatoria de derechos humanos. Bajo estas condiciones los tratados internacionales (en materia de derechos humanos) devenían insustanciales.

Este criterio se mantuvo y se reafirmó en diferentes formas a lo largo de la década de los noventa. No obstante, se modificó debido, entre otros factores, a la presión internacional y doméstica sobre la notoria falta de un compromiso auténtico por parte del Estado mexicano para otorgar efectividad a los compromisos internacionales adquiridos en materia de derechos humanos. Así en 1999 se adoptó un nuevo criterio, de acuerdo con el cual:

LOS TRATADOS INTERNACIONALES. SE UBICAN JERÁRQUICAMENTE POR ENCIMA DE LAS LEYES FEDERALES Y EN UN SEGUNDO PLANO RESPECTO DE LA CONSTITUCIÓN FEDERAL.

(...) esta Suprema Corte de Justicia considera que los tratados internacionales se encuentran en un segundo plano inmediatamente debajo de la Ley fundamental y por encima del derecho federal y el local. Esta interpretación del artículo 133 constitucional, deriva de que estos compromisos internacionales son asumidos por el Estado mexicano en su conjunto y comprometen a todas sus autoridades frente a la comunidad internacional, por ello se explica que el Constituyente haya facultado al presidente de la República a suscribir los tratados internacionales en su calidad de jefe de Estado y, de la misma manera, el Senado interviene como representante de la voluntad de las entidades federativas y, por medio de su ratificación, obliga a sus autoridades. Otro aspecto importan para considerar esta jerarquía de los tratados, es la relativa a que en esta materia no existe limitación competencia entre la Federación y las entidades federativas, esto es, no se toma en cuenta la competencia federal o local del contenido del tratado, sino que por mandato expreso del propio artículo 133 el presidente de la República y el Senado pueden obligar al Estado mexicano en cualquier materia, independientemente de que para otros efectos esta sea competencia

20 Tesis P.C/92, Pleno, Gaceta del Semanario Judicial de la Federación, Octava época, No. 60, diciembre de 1992, p. 27. 
de las entidades federativas. Como consecuencia de lo anterior, la interpretación del artículo 133 lleva a considerar en un tercer lugar al derecho federal y local en una misma jerarquía, en virtud de los dispuesto en el artículo 124 de la Ley fundamental, el cual ordena que "las facultades que no están expresamente concedidas por esta Constitución a los funcionarios federales, se entienden reservadas a los Estados". ${ }^{21}$ (...)

Como se puede observar, al sostener este criterio la Suprema Corte de la Nación daba un golpe de timón respecto de la tesis que se había sostenido tradicionalmente en México y que como ya se dijo dominó incluso hasta la década de los noventa. Aun cuando no se rompe con el dogma de la supremacía constitucional, lo cierto es que a partir de este nuevo criterio se desprende que al ser considerados en un plano normativo superior, el contenido normativo de los tratados internacionales pudieran ser utilizado para juzgar sobre la constitucionalidad de las leyes ordinarías, sean estas federales o locales.

Este criterio seguiría siendo utilizado en los siguientes años; así, en 2007 la Suprema Corte de Justicia de la Nación recurre al criterio de 1999, pero lo renueva en el sentido de reafirmar la importancia que tienen los compromisos internacionales adquiridos por el Estado mexicano, y la necesidad que todas las autoridades los respeten y acaten. Así, este cuerpo colegiado sostuvo que:

LOS TRATADOS INTERNACIONALES SON PARTE INTEGRANTE DE LA LEY SUPREMA DE LA UNIÓN Y SE UBICAN JERARQUÍCAMENTE POR ENCIMA DE LAS LEYES GENERALES, FEDERALES Y LOCALES. INTERPRETACION DEL ARTÍCULO 133 CONSTITUCIONAL.

La interpretación sistemática del artículo 133 de la Constitución Política de los Estados Unidos Mexicanos permite identificar la existencia de un orden jurídico superior, de carácter nacional, integrado por la Constitución Federal, los tratados internacionales y las leyes generales. Asimismo, a partir de dicha interpretación, armonizada con los principios de derecho internacional dispersos en el texto constitucional, así como con las normas y premisas fundamentales de esa rama del derecho, se concluye que los tratados internacionales se ubican jerárquicamente abajo de la Constitución Federal y por encima de las leyes generales, federales y locales, en la medida en que el Estado Mexicano al suscribirlos, de conformidad con lo dispuesto en la Convención de Viena Sobre el Derecho de los Tratados entre los Estados y Organizaciones Internacionales o entre Organizaciones Internacionales y, además, atendiendo al principio fundamental de derecho internacional consuetudinario "pacta sunt servanda", contrae libremente obligaciones frente a la comunidad internacional que no pueden ser desconocidas invocando normas de derecho interno y cuyo incumplimiento supone, por lo demás, una responsabilidad de carácter internacional. ${ }^{22}$

El siguiente paso, esto es, equiparar los tratados internacionales en derechos humanos a la Constitución, fue dado de forma clara en 2010 por el Primer Tribunal

21 Tesis P.LXXVII/99, Pleno, Semanario Judicial de la Federación y su Gaceta, Novena Época, Tomo X, Noviembre de 1999, Tesis aislada (constitucional).

22 Tesis P. IX/2007, Semanario Judicial de la Federación y su Gaceta, Novena Época, Tomo XXV, abril de 2007, p. 6. 
Colegiado en Materia Administrativa y de Trabajo del Décimo Primer Circuito, el cual llegó a sostener que:

\section{LOS TRATADOS INTERNACIONALES. CUANDO LOS CONFLICTOS SE SUS- CITEN EN RELACIÓN CON DERECHOS HUMANOS, DEBEN UBICARSE A NIVEL DE LA CONSTITUCIÓN.}

Los tratados o convenciones suscritos por el Estado mexicano relativos a derechos humanos, deben ubicarse a nivel de la Constitución Política de los Estados Unidos Mexicanos, porque dichos instrumentos internacionales se conciben como una extensión de lo previsto en esa Ley Fundamental respecto a los derechos humanos, en tanto que constituyen la razón y el objeto de las instituciones. Por lo que los principios que conforman el derecho subjetivo público, deben adecuarse a las diversas finalidades de los medios de defensa que prevé la propia Constitución y de acuerdo con su artículo 133 las autoridades mexicanas deben respetarlos, por lo que bajo ninguna circunstancia pueden ser ignorados por ellos al actuar de acuerdo a su ámbito competencial. ${ }^{23}$

Como se puede observar, en un lapso relativamente corto se gestó una nueva actitud de los tribunales federales a favor de los derechos humanos reconocidos a nivel internacional, ${ }^{24}$ lo cual en parte era resultado de una nueva generación de jueces que estaba intentando conseguir una revolución de los derechos, de tal manera que en un entorno más receptivo a los derechos humanos y en el que el papel de los interpreten de la constitución tuviera mayor peso, posiblemente ni siquiera hubiera sido necesaria una reforma constitucional como la que se está comentando; en el entendido que los tribunales ya estaban configurando la apertura del corpus de derecho internacional de los derechos humanos en el sistema jurídico mexicano.

\section{LA RESISTENCIA A LA REFORMA DE 2011 EN LOS CRITERIOS JURISPRUDENCIALES}

En vista de lo anterior, parecía razonable considerar que la publicación de la reforma constitucional en materia de derechos humanos acentuara esta tendencia protectora que se venía fraguando vía criterios jurisprudenciales. En ese sentido, lo cierto es que a partir de esa fecha (2011) ha sido copiosa la jurisprudencia que se ha emitido respecto a los alcances de la reforma y al significado de los

23 Tesis XI.1o.A.T.45 K, Semanario Judicial de la Federación y su Gaceta, Novena Época, Tomo XXXI, mayo de 2010, p. 2079.

24 Los tribunales colegiados de circuito se pueden considerar en el escaño inmediato inferior al de la Suprema Corte de Justicia dentro del organigrama del Poder Judicial de la Federación. Con el objetivo de lograr que la Suprema Corte se dedicara casi de forma exclusiva al control de la constitucionalidad, paulatinamente se han delegado muchas de sus facultades en los Tribunales Colegiados de Circuito, de manera que estos órganos se han convertido en la práctica en los más altos tribunales controladores de la legalidad en el país. Con todo, la Suprema Corte de Justicia ostenta una facultad de atracción, mediante la cual puede solicitar conocer de cualquier asunto que considere de trascendencia para la vida jurídica de la nación. De igual modo, en caso de controversia o contradicción de tesis entre Tribunales Colegiados, corresponde a la Suprema Corte dirimir dicha contradicción, estableciéndose de esta manera criterios obligatorios para todos los jueces y tribunales, mediante la llamada "contradicción de tesis". 
conceptos que se contienen en el artículo primero; destacando sobre todo la necesidad de que todas las autoridades y los jueces de todos los ámbitos deben llevar a cabo un control difuso de convencionalidad teniendo presente no sólo los tratados internacionales, sino también las resoluciones de órganos jurisdiccionales internacionales. ${ }^{25}$ Todas estas novedades hacían pensar a los observadores y sobre todo a la sociedad civil en un futuro promisorio para los derechos humanos en México, al menos en cuanto al papel de garante y promotor que estaba asumiendo la Suprema Corte de Justicia en particular, y los tribunales federales en lo general. Sin embargo, lo cierto es que la reforma en materia de derechos humanos ha desatado una serie de resistencias.

En el caso de la reforma en materia de derechos humanos esta resistencia ha llegado de distintos frentes. Así, por ejemplo, en enero de 2013 el diputado Francisco Arroyo Vieyra presentó una iniciativa para reformar nuevamente el artículo primero constitucional en el sentido de volver a imponer el principio de supremacía constitucional. ${ }^{26}$ De acuerdo con la exposición de motivos de dicha iniciativa, ${ }^{27}$ la redacción que resultó de la reforma constitucional de 2011 generó ambigüedad, pues de acuerdo con lo expresado por el diputado Vieyra, existe una contradicción entre el primero y el segundo párrafo; esto es, entre el principio de supremacía constitucional que se mantiene en el párrafo primero y el principio pro persona contenido en el segundo; lo cual, en opinión del autor de esa iniciativa, dificulta el trabajo de los jueces pues resta certeza acerca de cuál es el texto que debe prevalecer en cada caso. En entrevista ante los medios el diputado que promovía esta nueva reforma explicaba que no se trataba sólo una idea o capricho suyo, sino que era el resultado de pláticas sostenidas con jueces, magistrados, abogados litigantes y organizaciones civiles de defensa de los derechos humanos. Si bien la iniciativa no prospero, ya que desde que se hizo pública tuvo una dura recepción en la opinión pública y entre los defensores de los derechos humanos; lo cierto es que fue un claro intento regresivo, el cual que podía haber eliminado los avances obtenidos con la reforma de 2011.

Las declaraciones del diputado Arroyo Vieyra anunciaban la otra fuente de resistencia, esto es, la que se ha gestado entre jueces de todo tipo, incluidos los del Poder Judicial de la Federación y la propia Suprema Corte de Justicia. Estas resistencias no son expresas, ya que el ethos judicial obliga a los jueces a defender que actúan con apego a la constitución; sin embargo, las mismas se pueden inferir del contenido de las resoluciones que estos adoptan.

En relación a la Suprema Corte de Justicia, los mecanismos actuales de transparencia permiten dar seguimiento al sentido de votos que cada uno de los ministros emite en cada caso que les es sometido. De esta manera es posible apreciar cuál ministro mantiene consistentemente criterios favorables a los derechos humanos y

25 Jurisprudencia De La Corte Interamericana De Derechos Humanos. Es Vinculante Para Los Jueces Mexicanos Siempre Que Sea Más Favorable A La Persona. (Tesis P./J.21/2014, Gaceta del Seminario Judicial de la Federación, Décima época, Libro 5, abril de 2014, Tomo 1, p. 204).

26

27

Cfr., El universal, 03 de enero de 2013, http://archivo.eluniversal.com.mx/notas/893597.html

Consultable en http://archivo.eluniversal.com.mx/graficos/pdf13/iniciativa_reforma_articulo1.pdf 
cuales muestran diferentes formas de resistencia. A todo esto, hay que tomar en cuenta que un cambio de esta magnitud se opone a los principios que generaciones enteras de abogados consideraron como la base de su práctica, lo cual permite explicar en buena parte estos procesos de resistencia.

En esta tesitura, contrariando una tradición de ampliación de la esfera protectora de los tratados internacionales en materia de derechos humanos, una nueva configuración de la Suprema Corte, de tendencias menos progresistas, ha adoptados un par de criterios que no sólo pueden dar al traste con varias de décadas de adelantos jurisprudenciales, sino que apurando la situación, pone en jaque el futuro de la propia reforma.

En septiembre de 2013 la Suprema Corte de Justicia resolvió una contradicción de tesis de la que resultó el criterio que se transcribe a continuación, el cual, al tener el carácter de jurisprudencia, resulta de observancia obligatoria, de ahí su relevancia. De acuerdo con esta tesis:

LOS DERECHOS HUMANOS CONTENIDOS EN LA CONSTITUCIÓN Y EN LOS TRATADOS INTERNACIONALES. CONSTITUYEN EL PARÁMETRO DE CONTROL DE REGULARIDAD CONSTITUCIONAL, PERO CUANDO EN LA CONSTITUCIÓN HAYA UNA RESTRICCIÓN EXPRESA AL EJERCICIO DE AQUÉLLOS, SE DEBE ESTAR A LO QUE ESTABLECE EL TEXTO CONSTITUCIONAL.

El primer párrafo del artículo 1o. constitucional reconoce un conjunto de derechos humanos cuyas fuentes son la Constitución y los tratados internacionales de los cuales el Estado Mexicano sea parte. De la interpretación literal, sistemática y originalista del contenido de las reformas constitucionales de seis y diez de junio de dos mil once, se desprende que las normas de derechos humanos, independientemente de su fuente, no se relacionan en términos jerárquicos, entendiendo que, derivado de la parte final del primer párrafo del citado artículo 1o., cuando en la Constitución haya una restricción expresa al ejercicio de los derechos humanos, se deberá estar a lo que indica la norma constitucional, ya que el principio que le brinda supremacía comporta el encumbramiento de la Constitución como norma fundamental del orden jurídico mexicano, lo que a su vez implica que el resto de las normas jurídicas deben ser acordes con la misma, tanto en un sentido formal como material, circunstancia que no ha cambiado; lo que sí ha evolucionado a raíz de las reformas constitucionales en comento es la configuración del conjunto de normas jurídicas respecto de las cuales puede predicarse dicha supremacía en el orden jurídico mexicano. Esta transformación se explica por la ampliación del catálogo de derechos humanos previsto dentro de la Constitución Política de los Estados Unidos Mexicanos, el cual evidentemente puede calificarse como parte del conjunto normativo que goza de esta supremacía constitucional. En este sentido, los derechos humanos, en su conjunto, constituyen el parámetro de control de regularidad constitucional, conforme al cual debe analizarse la validez de las normas y actos que forman parte del orden jurídico mexicano. ${ }^{28}$

La promoción en la que se denunciaba la contradicción de tesis le fue turnada al ministro Arturo Zaldívar Lelo de Larrea, quien formuló un primer proyecto

28 Tesis P.J.20/2014, Gaceta del Semanario Judicial de la Federación, Décima Época, Libro 5, abril de 2014, Tomo I, p. 202. 
en el que defendía la prevalencia del principio pro-persona, aun en detrimento de las disposiciones constitucionales que impusieran restricciones. Ese primer proyecto el ministro Zaldívar, consideraba que en caso de contradicción entre un tratado internacional y la Constitución, cuando esta impone de forma expresa alguna restricción, debía prevalecer el tratado internacional. Este proyecto fue votado en contra por todos los ministros, excepto por José Ramón Cossío Díaz quien votó a favor. Ante la negativa, el ministro Arturo Zaldívar reformuló su proyecto en el sentido que le indicaba la mayoría. Sometido nuevamente a votación, todos los ministros votaron a favor de la nueva redacción excepto el ministro Cossío Díaz, quien voto en contra. ${ }^{29}$

Esta tesis pretende atenerse a una interpretación literal del texto constitucional, aun cuando en realidad parte de una noción que no acepta la importancia de los tratados internacionales dentro del sistema jurídico mexicano, pese a que es el propio Estado mexicano el que de manera soberana decide suscribirlos. En realidad este criterio representa la anulación ad hoc de los derechos humanos consagrados en tratados internacionales, bajo el argumento de que la Constitución establece limitantes en un determinado sentido. Significa también que llegado el caso se puede dejar sin efectos el principio pro persona, ya que en efecto la Constitución contiene diversos supuestos de restricción a derechos humanos, y lo que es peor, futuras reformas constitucionales pudieran introducirle otros más. En términos breves, esta jurisprudencia echa por el suelo la gran ventaja de la reforma de 2011, tal como fue redactada, esto es, que obligaba al intérprete a optar siempre por la interpretación que más beneficiara a la persona, ahora ya no es así. Criterios posteriores han querido hacer hincapié en la importancia del principio pro persona, sin embargo, al no oponerse de manera expresa a la tesis de las restricciones constitucionales, dejan subsistente esta última.

Por supuesto, dicha tesis fue recibida con recelo por parte de las organizaciones civiles defensoras de los derechos humanos, las cuales la supieron ver como un claro intento por bloquear los avances logrados con la reforma de $2011 ; 30$ en otras palabras, la consideraron como un criterio regresivo. Para los defensores de los derechos humanos la reforma al artículo primero constitucional implicaba que la decisión de reconocer o no un derecho dejaba de estar sujeta del parecer de las autoridades mexicanas y pasaba a depender del reconocimiento de instancias internacionales, lo cual representaba una muy clara señal de que la lucha por los derechos podía consolidarse. Por el contrario el criterio jurisprudencial transcrito confirma a México como un país reacio a respetar los derechos humanos reconocidos en sede internacional.

29 Debido a la importancia que tuvo esta resolución, diversos medios siguieron con interés el desarrollo de los debates, los cuales se prolongaron por varios días. Como referencia se pueden consultar Cima Noticias de 27 de agosto de 2013 (https://cimacnoticias.com.mx/node/64101) y Excélsior de 29 de agosto (https://www. excelsior.com.mx/nacional/2013/08/29/916181).

30 Una carta pública titulada "La reforma constitucional en riesgo" fue dirigida a los ministros de la Suprema Corte, la cual fue firmada por reconocidos académicos y decenas de organizaciones defensoras de los derechos humanos. La misma puede consultarse en: http://cmdpdh.org/wp-content/uploads/2013/09/Comunicado-finaldiscusiones-SCJN.pdf 
A continuación se transcribe otra tesis jurisprudencial que ya es obligatoria y que también debe ser considerada como una clara muestra de resistencia a la reforma; además, es un claro ejemplo de que el marco teórico subyacente en la redacción anterior del artículo primero puede imponerse al actual. Cierto que el propio numeral permite la posibilidad si es que se recurre a una interpretación textual, pero si se le interpreta echando mano de las reglas que se contienen en el mismo, entonces, la interpretación correcta implicaría hacer prevalecer el principio pro persona. A continuación se transcribe el criterio en cuestión, posteriormente se desahogan algunos comentarios.

\section{DERECHOS HUMANOS Y SUS GARANTÍAS. SU DISTINCIÓN}

Antes de las reformas constitucionales de 6 y 10 de junio de 2011, las voces "derechos humanos y sus garantías", eran términos que solían confundirse, ambigüedad que posiblemente derivaba de la anterior denominación del Capítulo I de la Constitución Política de los Estados Unidos Mexicanos, titulado "De las garantías individuales". Sin embargo, el Poder Reformador de la Constitución, con las citadas reformas, elevó a rango constitucional su distinción, como deriva de las siguientes menciones: i) el capítulo I cambió su denominación a "De los derechos humanos y sus garantías"; ii) en el artículo 1o se especificó que todas las personas gozaran de los derechos humanos reconocidos en la Constitución y en los tratados internacionales "así como de las garantías para su protección", y iii) en el numera 103 fracción I, se especificó que los Tribunales de la Federación resolverán toda controversia que se suscite por infracción a los derechos humanos y las garantías otorgadas para su protección". Luego, para el Constituyente Permanente los "derechos y sus garantías" no son lo mismo, ya que éstas se otorgan para proteger los derechos humanos; constituyen, según Luigi Ferrajoli, los "deberes consistentes en obligaciones de prestación o en prohibiciones de lesión, según que los derechos garantizados sean derechos positivos o derechos negativos", es decir, son los requisitos, restricciones, exigencias u obligaciones previstas en la Constitución y en los tratados destinadas e impuestas principalmente a las autoridades, que tienen por objeto proteger los derechos humanos; de ahí que exista una relación de subordinación entre ambos conceptos, pues las "garantías" sólo existen en función de los derechos que protegen; de tal suerte que pueden existir derechos sin garantías pero no garantías sin derechos. Así, a manera de ejemplo, puede decirse que el derecho humano a la propiedad tienen, entro otras garantías, las de audiencia y legalidad, pues prohíbe a la autoridad molestar a un particular sin mandamiento escrito en el que funde y motive la causa legal del procedimiento, y que los gobernados sean privados de la propiedad sin previa audiencia. ${ }^{31}$

Curiosamente, el mismo tribunal colegiado que emitió el criterio anterior, había sostenido una tesis diferente al interpretar del artículo 107 fracción V de la

31 Gaceta del Semanario Judicial de la Federación, Libro 17, abril de 2015, Tomo II, tesis XXVII. 3o. J/14, Décima época. Jurisprudencia Constitucional. Por otra parte, de forma recientemente en 2018 la Segunda Sala de la Suprema Corte de Justicia ha adoptado un criterio similar, lo cual no hace sino confirmar esta forma fundamentalista de entender los derechos humanos y de entronizar esta distinción, que como ya se expresó, resulta cuando menos inconveniente y contraria al principio pro persona. (Cft. DERECHOS HUMANOS Y SUS Garantías. SuS Diferencias, Semanario Judicial de la Federación, Segunda Sala, Tesis aislada, 2a LXXXVIII/2018 (10a), No. Registro 2017890.). 
Ley de Amparo, en la cual sostenía que tanto los derechos humanos como sus garantías deben considerarse como derechos sustantivos, es decir, que no se puede distinguir ni jurídica ni conceptualmente entre unos y otras; ${ }^{32}$ no obstante, posteriormente modificó su criterio para ajustarlo al que ya se había sostenido con anterioridad en cuatro ocasiones por otros tribunales; con lo que una vez sumada la quinta resolución en el mismo sentido (es decir la que se acaba de transcribir), dicha tesis pasó a ser de aplicación obligatoria.

En efecto, desde la perspectiva que se ha adoptada en este trabajo, conforme a la cual el principio pro persona debería prevalecer en todo momento, no existe posibilidad de distinguir sustantivamente entre derechos y garantías para su protección, entre otras razones por que un derecho no es simplemente la expresión escueta contenida en una expresión como por ejemplo "todo individuo tiene derecho a la libertad" (artículo 3 de la Declaración Universal de los Derechos humanos), sino que en realidad el derecho está compuesto por todos aquellos instrumentos normativos que le permitan hacerse operacional. El ejemplo que pretenden utilizar en las últimas líneas de la jurisprudencia que se transcribe es claro ejemplo de ello, ya que se pretende argumentar que el derecho de propiedad tiene como garantías a la de audiencia y legalidad, cuando estos dos últimos son por sí mismos auténticos derechos humanos, reconocidos tanto en tratados internacionales como en la Constitución. De manera que en un sentido cabal, no hay lugar para la distinción que se pretende establecer con el criterio jurisprudencial en comento.

La consecuencia lógica de un criterio como el que terminó por imponerse, es que abre la posibilidad de concebir derechos de primera clase o auténticos derechos humanos, frente a derechos de segunda o simples mecanismos de protección (garantías). ${ }^{33}$ Lo cierto es que, tal como se ya apuntó en el tercer apartado de este trabajo, la reforma al artículo primero constitucional no eliminó por completo la referencia a las garantías, sino que tan sólo la sustituyó como protagonista del texto. Más allá de la razón por la cual se conservó en el texto constitucional la referencia al termino garantías, lo cierto es que una cuestión que en un principio bien pudo haberse considerado meramente teórica, terminó por ser motivo de una situación bastante practica; que ha generado consecuencias en casos concretos y que resulta de observancia obligatoria para otros casos, por lo cual puede afectar a un número indeterminado de personas en un futuro.

32 Derechos sustantivos. Por este concepto contenido en el artículo 107, fracción V, de la Ley de Amparo, no sólo deben considerarse los derechos humanos sino también las garantías contenidas en el llamado Parámetro de Control de la Regularidad Constitucional (Tesis XXVII.3o. 60 K (10a), Gaceta del Semanario Judicial de la Federación, Décima Época, Libro 10, septiembre de 2014, Tomo III, p. 2392)

33 Este criterio responde a una postura iusfilosófica fundamentalista propiamente iusnaturalista. Estas doctrinas consideran que existe un derecho ideal y que este responde a un fundamento absoluto, ya sea la Razón, la Revelación o la Naturaleza Humana. Por el contrario, desde una postura naturalizada (que no iusnaturalista) se renuncia a la necesidad de fundamentar filosóficamente le concepto de derechos humanos y por lo tanto también se elimina la posibilidad de calificar exigencias determinadas como auténticos derechos humanos frente a otros que no lo son. Desde una postura naturalizada lo más que se puede afirmar es que los derechos reconocidos a nivel internacional son derechos humanos y que por concesión soberana merecen la mayor tutela y protección en el derecho interno (Cfr. RABOssi, "Eduardo La teoría de los derechos humanos naturalizada", en Revista del Centro de Estudios Constitucionales, No. 5, enero-marzo de 1990, 159-175.) 
Claramente se trata de un criterio regresivo, que si bien está asentado en el texto mismo, en realidad muestra una interpretación propia del estado de cosas anterior a la reforma. Una vez más, en caso de que los tribunales hubieran optado por una interpretación progresista, más acorde con la pretensión de imponer un nuevo paradigma, hubieran tenido la posibilidad de haberlo hecho, utilizando como fundamentación el principio pro persona contenido en el párrafo segundo del mismo artículo primero.

El grave problema que surge es que al utilizar esta jurisprudencia, los jueces ordinarios tendrán que decidir cuáles de los derechos previstos en la constitución y en los tratados internacionales son auténticos derechos o derechos principales y cuáles son meras garantías. Como se puede apreciar es una gran ventana que se abre para otras interpretaciones restrictivas. Lo cierto es que una interpretación progresista, ajustada a las posibilidades interpretativas del principio pro persona, en la que realmente se quisiera hacer valer un bloque de constitucionalidad, no estaría interesada en hacer distinción alguna entre auténticos derechos humanos y simples garantías, sino que se inclinaría simplemente por aceptar un bloque de derechos constitucionales y convencionales. Una interpretación como esta potenciaría la protección a las personas y fortalecería la posibilidad real del bloque unificado de constitucionalidad y convencionalidad que se buscaba crear con la reforma al artículo primero constitucional.

\section{CONSIDERACIONES FINALES}

Antes que nada es justo reconocer la trascendencia de la reforma que se ha comentado a lo largo de este trabajo. En sí misma es uno de los mayores intentos que se han hecho para modificar pautas arraigadas en el sistema jurídico mexicano y en la forma en la que lo entienden quienes lo operan. Esta modificación se originó en medio de una grave crisis de violencia y de credibilidad del Estado. Esto ha provocado que al menos el ciudadano de a pie posiblemente no sólo no aprecie la trascendencia de la misma, sino que no haya logrado observar cambio alguno en las pautas cotidianas de violación sistemática a derechos que se padecen en las últimos años.

En cambio, para los operadores del sistema la reforma tuvo un enorme significado, (incluyendo aquí no sólo a jueces, funcionarios públicos y abogados, sino también a todos aquellos interesados en la promoción y protección de los derechos humanos); en efecto, se esperaba que provocara un cambio profundo en las prácticas jurídicas y en la forma en la que se entiende el derecho en este país. Ahora bien, determinar hasta dónde se ha operado esta transformación, es una cuestión complicada. Como se ha logrado observar a lo largo de este trabajo, al menos para el caso de los tribunales federales, incluida la Suprema Corte de Justicia, se está ante un panorama de claroscuros.

Por una parte es conveniente aceptar que se ha hecho un esfuerzo enorme por promover y socializar la reforma y por capacitar a los operadores en el nuevo marco teórico. Pero por otra, las inercias y las resistencias, conscientes o inconscientes, 
siguen manifestándose de forma meridiana. Muestra de ello son los criterios adoptados recientemente por los tribunales federales, algunos de los cuales, como se pudo observar, representan un giro radical en relación a la tradición de defensa y promoción que había caracterizado a los criterios jurisprudenciales de los años previos a la reforma. Justamente esos criterios de finales de los años 90 y los primeros años del nuevo siglo, fueron uno de los promotores del cambio. Más aún, bajo una interpretación generosa se puede decir que algunos de estas tesis jurisprudenciales hacían ociosa la reforma; es decir, a través de estos criterios, un intérprete comprometido con la defensa de los derechos podía hacer uso del bloque de tratados en materia de derechos humanos y hacerlos valer como argumento para sustentar una resolución o una petición. Sin embargo, en un contexto que de suyo se ha mostrado refractario a la idea de derechos humanos, no sólo no se sacó el provecho debido de los criterios jurisprudenciales que los promovía; sino que, ante la falta de resultados se hizo necesario promover una reforma constitucional para hacer explícito lo que ya se desprendía de interpretaciones progresistas.

Como se puede colegir de lo dicho en este trabajo, la reforma en sí misma no puede verse como un cambio de paradigma, sino como un mero precursor, que desafortunadamente no ha logrado generar los frutos que se esperaban. Cuando se les pregunta, por regla general los operadores del sistema jurídico afirman que ya están aplicando el nuevo marco teórico con todas sus consecuencias; sin embargo, de lo que se pudo observar en el análisis de los criterios jurisprudenciales revisados, son evidentes los procesos de resistencia que se están generando. Estas inercias pueden ser inconscientes, debido a que buena parte de los operadores han sido educados bajo el anterior paradigma, y buena parte de su carrera profesional han aplicado un marco teórico distinto. De manera que es muy probable que la resistencia no sea consciente, sino que sea reflejo de una fuerte formación dentro del anterior paradigma. De no ser así, deberíamos considerar que el clientelismo, el autoritarismo y la falta de independencia de los tribunales y el poder legislativo (respecto del ejecutivo) están distorsionando la aplicación de la reforma.

Lo grave en este proceso es que en el caso de los tribunales federales y la Suprema Corte de Justicia, se trata de operadores privilegiados, cuya opinión debe ser tomada en cuanta por el resto de los usuarios del sistema. En otras palabras, estos actores pueden por sí mismos detener el avance de la reforma o bien pueden potenciarla. Hasta ahora, al menos en la actual configuración de la Suprema Corte, todo parece indicar que existe una tendencia hacia los criterios restrictivos. Mientras esto suceda la reforma constitucional corre el riesgo de transformarse en letra muerta.

Con todo, de la propia redacción del artículo primero se desprende la posibilidad de que en una interpretación generosa de los derechos humanos, jueces menores, o cualquier autoridad del estado, asuman la decisión de emitir resoluciones progresistas (garantistas), aún en contra de lo que se estipula en la jurisprudencia. Sin embargo, es preciso reconocer que no existen incentivos para que los operadores jurídicos de menor jerarquía actúen de este sentido. Por el contrario, 
la regla es que ajusten sus opiniones a lo que se expresa en los criterios de los tribunales superiores, asegurando de ese modo la pertinencia formal de lo que se exprese en sus resolutivos. En cualquier caso, la parte más grave es que abre la posibilidad para que se adopten otros criterios restrictivos a futuro, según se puede apreciar de lo dicho en las líneas que anteceden.

A modo de conclusión, se puede considerar que ni la reforma constitucional, ni los criterios adoptados por la actual configuración de la Suprema Corte de Justicia, pueden ser vistos como precursores de una "revolución de los derechos" en el sistema jurídico mexicano. En ese sentido, el cambio de paradigma que se esperaba sucediera como consecuencia de la reforma, aún no se ha concretado. Ni siquiera si se considerara el sistema jurídico como el cuerpo normativo y nada más; mucho menos si se le entiende como el conjunto de prácticas que se asientan en dicho marco normativo y teórico.

Hacer el seguimiento de las resoluciones de los tribunales federales es asequible en la medida de que existen los medios institucionalizados de publicación de las resoluciones de estos órganos jurisdiccionales, ahora incluso, disponibles en formato electrónico. Sin embargo, esta facilidad no es la misma cuando se intenta conocer las resoluciones de los jueces ordinarios, autoridades administrativas, fiscalías, y agencias estatales de seguridad (incluidas en estos momentos las fuerzas armadas). En todo caso, el siguiente paso en la valoración del avance de la reforma, es conocer la manera en la los operadores jurídicos en los escaños inferiores del aparato judicial, así como las autoridades administrativas, están aplicando la reforma en cuestión.

\section{BIBLIOGRAFÍA}

AYALA CORAO, Carlos M., La jerarquía constitucional de los tratados relativos a derechos humanos y sus consecuencias, Colegio de Secretarios de la Suprema Corte de Justicia de la Nación, México, 2003.

CABALLERO OCHOA, José Luis, "La cláusula de interpretación conforme y el principio pro persona (artículo primero segundo párrafo de la constitución)”, en Carbonell Sánchez, Miguel y Salazar Ugarte, Pedro (coords.) La reforma constitucional de derechos humanos: un nuevo paradigma, UNAM-IIJ, México, 2011, pp. 103-133, https://archivos.juridicas.unam.mx/www/bjv/libros /7/3033/6.pdf.

CARREÓN GALLEGOS, Ramón Gil, "Derechos humanos, garantías individuales y derechos fundamentales. Problema terminológico o conceptual, en Cienfuegos Salgado David y Froto Mandariaga German (Coords.), Los derechos humanos en el momento actual, Comisión de Derechos Humanos de Coahuila, México, 2012, pp. 131-145, https://archivos.juridicas.unam.mx/www/bjv/ libros/7/3171/7.pdf.

Comisión Interamericana de Derechos Humanos, Situación de derechos humanos en México, 2015, http://www.oas.org/es/cidh/informes/pdfs/mexico2016-es.pdf. 
CRUZ PARCERO, Juan Antonio, Hacía una Teoría Constitucional de los Derechos Humanos, Instituto de Estudios Constitucionales del Estado de Querétaro, México, 2017, https://archivos.juridicas.unam.mx/www/bjv/libros/ 10/4746/12.pdf

ENRÍQUEZ SOTO, Pedro Antonio, "La interpretación conforme y su impacto en los jueces mexicanos", en Serna de la Garza José María (Coord.), Contribuciones al derecho constitucional, UNAM, IIJ, México, 2015, pp. 289-215, https://archivos.juridicas.unam.mx/www/bjv/libros/8/3980/40.pdf.

EPP, Charles, La revolución de los derechos. Abogados, activistas y cortes supremas en perspectiva comparada, Siglo XXI, México, 2013.

FERRER MAC-GREGOR, Eduardo, "Interpretación conforme y control difuso de la convencionalidad. El nuevo paradigma para el juez mexicano", en CARBONELL SÁNCHEZ, Miguel y SALAZAR UGARTE, Pedro (Coords.) La reforma constitucional de derechos humanos: un nuevo paradigma, UNAM-IIJ, México, 2011, pp. 339-429, https://archivos.juridicas. unam.mx/www/bjv/libros/7/3033/14.pdf.

GANDARIA Manrique, "CNDH recaba 20 mil quejas en contra de Ejercito Marina y PF", en El Sol de México, 12 de noviembre de 2017, https:/www.elsoldemexico.com.mx/mexico/justicia/cndh-recaba-20-mil-quejas-en-contradel-ejercito-marina-y-pf-311915.html.

HABERMAS, Jürgen, Facticidad y validez. Sobre el derecho y el estado social de derecho en términos de teoría del discurso, Trotta, Madrid, 4a ed. 1998, pp.

LABARDINI, Rodrigo, "Proteo en México. Un nuevo paradigma: derechos humanos y constitución", en Boletín Mexicano de Derecho Comparado, No. 133, enero-abril de 2012, pp. 319-353. https://revistas.juridicas.unam.mx/ index.php/derecho-comparado/article/view/4743/6094

RABOSSI, "Eduardo La teoría de los derechos humanos naturalizada", en Revista del Centro de Estudios Constitucionales, No. 5, enero-marzo de 1990, 159175, https://dialnet.unirioja.es/servlet/articulo?codigo=1049162

RAWLS, John, Teoría de la justicia, Fondo de Cultura Económica, México, 2010. VÁZQUEZ, Luis Daniel y SERRANO, Sandra, "Los principios de universalidad, interdependencia, indivisibilidad y progresividad. Apuntes para su aplicación práctica”, en Carbonell Sánchez, Miguel y Salazar Ugarte, Pedro (Coords.) La reforma constitucional de derechos humanos: un nuevo paradigma, UNAMIIJ, México, 2011, pp.135-165, https://archivos.juridicas.unam.mx/www/bjv/ libros/7/3033/7.pdf. 